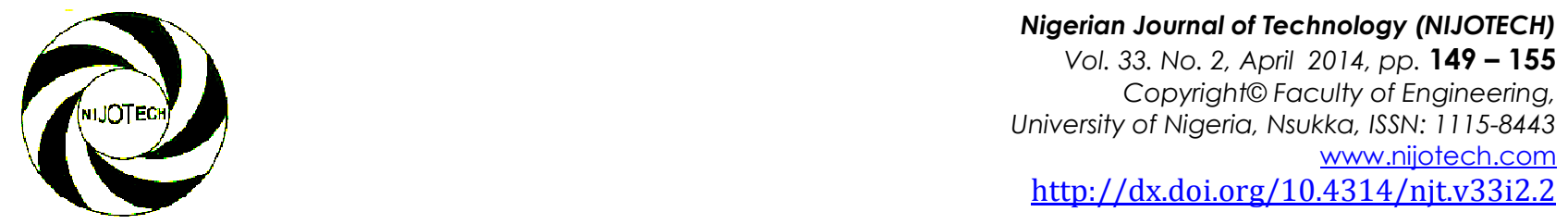

\title{
PERFORMANCE ANALYSIS OF AN EXPERIMENTAL CENTRIFUGAL PUMP
}

\author{
A. A. Adeniyi ${ }^{1}{ }^{*}$, O. D. Komolafe ${ }^{2}$ \\ 1DEPARTMENT OF MECHANICAL ENGINEERING,UNIVERSITY OF ILORIN, NIGERIA. \\ 2DEPARTMENT OF MEChANICAL \& MECHATRONICS ENGINEERING, FEDERAL UNIVERSITY OYE-EKITI, NIGERIA.
}

E-mail addresses: ${ }^{1}$ adeniyi.aa@unilorin.edu.ng 2 daniel.komolafe@fuoye.edu.ng

\begin{abstract}
This paper presents results on the performance characteristics of an experimental H47-centrifugal pump. Four dimensionless numbers, namely Reynolds, flow coefficient, head coefficient and power coefficient are used. The pump was operated nominally at Reynolds number of $4 \times 10^{7}, 3.4 \times 10^{7}$ and $2.7 \times 10^{7}$ respectively. The performance charts show that the maximum possible efficiency of the pump is less than $45 \%$ and the best efficiency point is around $42 \%$. The maximum possible power coefficient is $2.48 \times 10^{-4}$ at a corresponding flow coefficient of $3.2 \times 10^{-3}$ giving a head coefficient of 0.078 at the best efficient point (BEP). Electronic sensors on the $H 47$ rig provided measurements that ensured noticing of fluctuations across the Venturi meter caused by the onset of cavitation.
\end{abstract}

Keywords: centrifugal pump $H 47$ rig, pump performance, BEP.

\section{INTRODUCTION}

Centrifugal pumps are employed in a wide range of engineering applications. They find applications in pumping of liquids like water and oil. They are also applied in pumping of slurries, such as cement. The pump assembly often consists of an electric motor that creates the rotary motion required to turn the spiral impellers. The centrifugal pump is robust, effective and relatively inexpensive to operate and maintain. The fluid enters the pump axially through the suction pipe via the eye of the impeller. The fluid flows from the inlet to the impeller centre and is discharged radially from the impeller out along its blades around the entire circumference. The flow is directed either into a ring of stationary diffuser vanes and through them into the volute casing or directly into the casing. The casing collects the fluid, decelerates it, thus converting some of the kinetic energy into pressure energy and finally discharges the fluid through the delivery flange. The centrifugal force thereby increases the fluid velocity and consequently also transforms the kinetic energy to pressure head. In other words, the pump converts mechanical energy to hydraulic energy of the fluid to get the fluid to the required height through the centrifugal force of the impeller blade. For industrial or home applications, pump of sufficient head is required to take water from a well to the overhead tank. The well is usually below the tank. Once the water is taken to the tank level, the flow can serve the users by flowing under gravity and no pump will be required. In the centrifugal pump, the pumping action is created by suction. There are a number of possible classifications for pumps. The classification can be based on their purpose, the construction material, the fluid that is pumped, the orientation of the pumps or the way energy is added to the system [1]. Based on the energy method classification, there are kinetic and positive displacement pumps. Rotary and reciprocating pumps are positive displacement pumps. Special effect, regenerative turbine and centrifugal pumps are the kinetic pump category.

The H47 centrifugal pump test set was used for this experiment. H47 was developed by TecQuiptment Ltd., Long Eaton, Nottingham UK [2] as teaching equipment for studying pump flow. It was designed to deliver $120 \mathrm{kPa}$ (or $12.2 \mathrm{~m}$ ) maximum head at a maximum speed of $3000 \mathrm{rpm}$ and a maximum flow rate of $2 \mathrm{~L} / \mathrm{s}$. A $500 \mathrm{~W}$ single phase motor powers the pump. The H47 experimental test set consists of a mechanical section and an electronic sensor readout section, where data are read. The impeller type is the semi-open impeller type [1]. The blades can be viewed from a glass as shown in Figure 1. In Figure 1(B), the

* Corresponding author, Tel: $+234-8032820144$ 
impeller can be seen when the stroboscope light is on and set to the frequency (angular velocity) of the shaft rotation. In Figure 1(C), the cavitation or formation of bubbles can be seen well in the black and white format enhancement.

Dimensionless numbers are of key importance in parametric analysis of engineering problems. Their use allows the important task of data reduction of similar problems and useful to predict changes in the performance characteristics of an existing pump or to estimate the performance of a modified pump design prior to the construction of a prototype. An engineer choosing a new pump or testing an existing pump to do a certain job, can save time and money by testing on a small pump to estimate the performance of an identical but larger pump. The engineer can then use dimensional analysis and similarity laws to reduce the amount of measurements he needs and to provide a compact representation of the results. For this to be the case the coefficients (dimensionless numbers) must have the same values for each size of the pump.

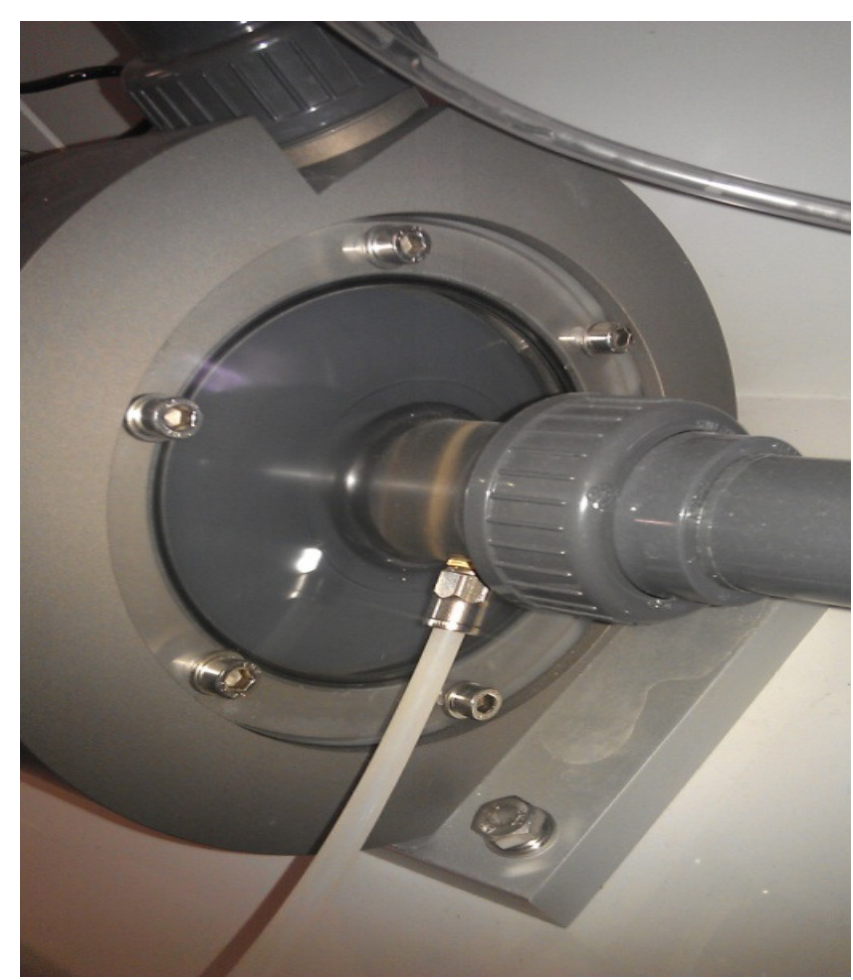

(A) Without stroboscope

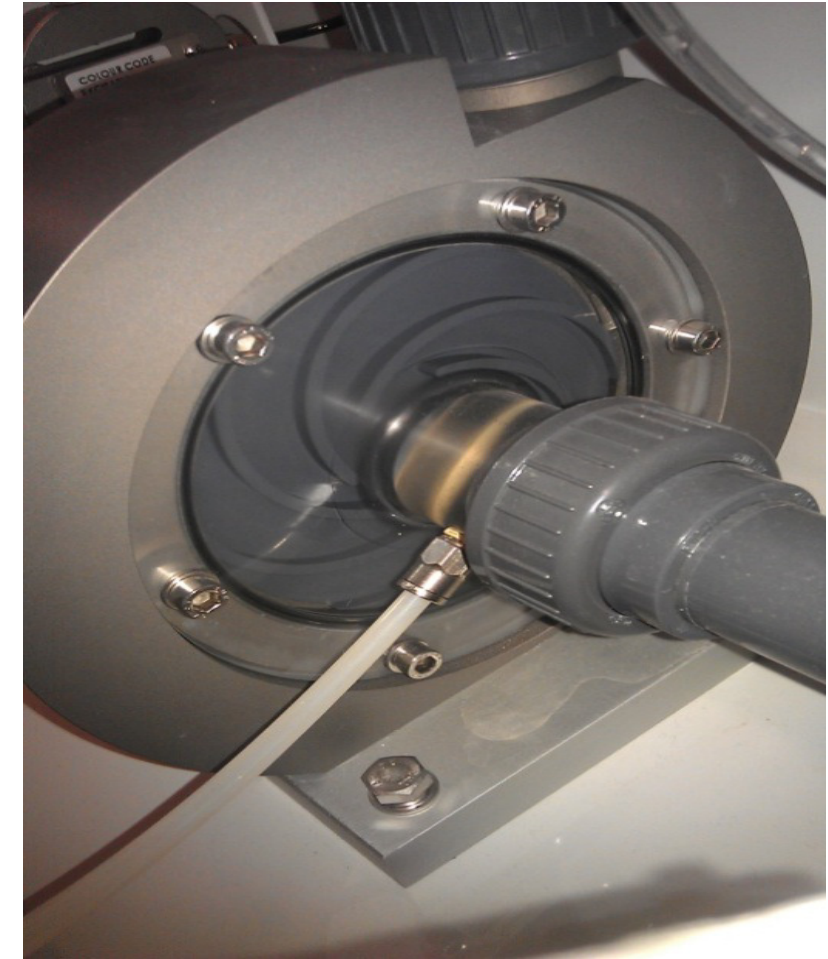

(B) Stroboscope set to motor frequency

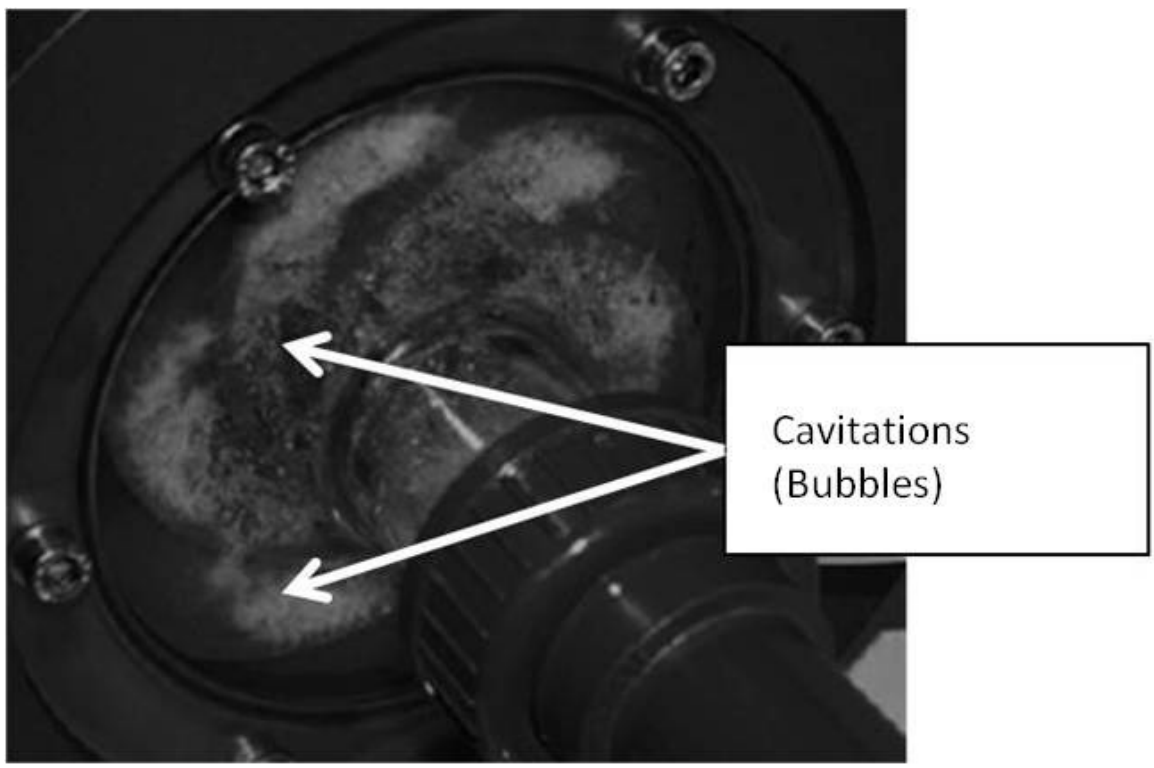

(C) Operating during cavitation with the stroboscope on

Figure 1: Selected photographs of the pump showing the blades during operation 
The H47 rig is popularly used in fluid mechanics laboratories but there are hardly any good reference materials available on this students' experimental rig. This paper presents data and a simplified analysis of the rig. In this work the $\mathrm{H} 47$ centrifugal pump characteristics were treated in a dimensionless form. Pump characteristics curve makes it possible to use only one characteristic curve for all possible operating speeds to be tested [3]. A number of researchers [4-6] have taken on the task of performance analysis and sizing of centrifugal pumps in attempt to raise the efficiency of this kind of machinery Ozor \& Ojobor [4] for example, designed, constructed and carried out experiments to measure the performance of a singleentry centrifugal pump demonstration unit. Their instrumentation was mechanical. In the work, they found that the tested pump can develop a maximum head and flow of $18.35 \mathrm{~m}$ and 21.4 litres per minute respectively at a maximum speed of 2,800 rpm and input power of 370 watts. In this work, electronic sensors and instrumentation give the added advantage of better measurements. With which it was possible to observe the fluctuations better.

\section{FUNDAMENTALS OF THE PUMP FLOW}

\subsection{Governing equations}

The basic equations describing the flow of pump are given in this section. For simplicity, the transient dynamics of the fluid has been described.

\subsubsection{Flow rate}

The flow rate, $Q$, is calculated from the pressure drop along the Venturi, by using equation (1).

$Q=C_{d} A_{1} \sqrt{\frac{2 \Delta p}{\rho\left(\frac{A_{1}^{2}}{A_{2}^{2}}-1\right)}}$

\subsubsection{Pump Performance}

The performance of the centrifugal pump can be estimated using the reduced form of the steady flow energy equation (SFEE), equation (2). This equation indicates that the work done upon the fluid by the pump, ignoring kinetic energy and the difference in levels between pump inlet and outlet, is equal to the power input of the pump less any losses.

$Q\left(p_{0}-p_{1}\right)=P-L$

\subsubsection{Head}

The pump total head is calculated using the Bernoulli's equation (3). The pump provides the driving power to take water from the reservoir to the destination. In this case the reservoir and the destination are the same.

$\frac{p}{\rho}+\frac{v^{2}}{2}+g z=C$

The pump total head, $\mathrm{H}(\mathrm{m})$, is therefore as represented in equation (4). Head is also, often, referred to in units of pressure by multiplying it by $\rho g$.

$H=\frac{p_{0}-p_{1}}{\rho g}$

\subsubsection{Power input}

The input power from the motor is given by equation (5), where the torque, T, is obtained from the torque sensor and $\omega$ is the shaft speed.

$P=\omega T$

Hydraulic Power

The hydraulic power, otherwise also called the "water horsepower", of the pump is given by equation (6).

$P_{w}=\left(p_{0}-p_{1}\right) Q$

\subsubsection{Efficiency}

The overall efficiency of the pump is given by equation (7).

$\eta=\frac{P_{w}}{P}$

\subsection{Similarity laws}

The performance of geometrically similar machines, i.e. machines belonging to one family, is governed by similarity laws and may be represented for the whole family by a single plot of dimensionless characteristics. The Buckingham $\pi$ theorem is used to derive the relevant dimensionless characteristics, equations (9) - (13), following from equation (8).

$$
\begin{aligned}
& \pi_{1}=\frac{Q}{N D^{3}} \\
& \pi_{2}=\frac{g(\Delta H)}{N^{2} D^{2}} \\
& \pi_{3}=\frac{P_{w}}{\rho N^{3} D^{5}} \\
& \pi_{4}=\frac{N Q^{\frac{1}{2}}}{(\Delta H)^{\frac{3}{4}}} \\
& \pi_{5}=\frac{\mu}{\rho N D^{2}}
\end{aligned}
$$$$
F(Q, g \Delta H, \rho, \mu, \eta, D, N)=0
$$

These numbers $\left(\pi_{1}-\pi_{4}\right)$ are respectively the flow coefficient, $C_{Q}$, head coefficient, $C_{H}$, power coefficient, $C_{P}$, and specific speed, $N_{S} . \pi_{3}$ is also commonly referred to as the Newton number [3]. The reciprocal 
value of the dimensionless number $\pi_{5}$ is in the literature [3] the well-known Reynolds number. The characteristic length scale, $\mathrm{D}$, is taken as the impeller diameter. The specific speed is a parameter used for pumps and turbines to determine the best design to match a given pumped system. It follows that for a given speed, the specific speed is large for large flows and low heads and small for small flows and high heads. The important value is the one that corresponds to the conditions that produce the greatest efficiency. $N_{s}$ value may be calculated using the flow and head corresponding to the maximum efficiency [3].

\section{THE EXPERIMENTAL SETUP AND OPERATING PROCEDURE}

The experimental rig consists of the pump, the water reservoir and the electronic display panel as shown with a line diagram in Figure 2. The electronic sensors of the $\mathrm{H} 47$ rig are used to take the pressure, torque and power measurements. The $\mathrm{H} 47$ experimental test rig is a compact, mobile and fully self-contained centrifugal pump test set that allows students to find the characteristics of a centrifugal pump.

The measurements taken are designed to be able to measure flow rate, power input, hydraulic power and efficiency of the pump. The necessary measurements to be taken are decided based on the Buckingham $\pi$ parameters. The density, $\rho$, and the dynamic viscosity, $\mu$, of water are respectively taken to be $998 \mathrm{~kg} / \mathrm{m}^{3}$ and $9.6 \times 10^{-4}$ Pa.s. The transparent covering of the pump house makes it easy to see cavitation, but with the aid of a stroboscope.

The pressure drop readings are taken across the Venturi meter upstream of the flow. These are used to compute the flow rate as given in equation (1). The torque is measured using a strain gauge sensor. This sensor is connector to the electronic display which also displays the fluctuating shaft's speed and true shaft power. The reservoir is a simple container placed below the rig. The water is taken from the reservoir and returned to the same place ensuring a minimum consumption of water. The valve closing and opening creates different operating conditions.

The sensitivity of the instruments was carefully taken into consideration for taking the readings. The motor speed readings were within $\pm 1 \%$ of the speeds. The minimum pressure change below which the readings were not accurate is 0.05 bars. The speed of the motor fluctuates and there was need to re-adjust it using the speed control knob to maintain the $\pm 1 \%$ margin. In this work, 2000, 2500 and $3000 \mathrm{rpm}$ speeds were used. For each speed, the pump is operated until cavitation is noticed.

Figure 3, shows the Venturi section. The coefficient of discharge, $C_{d}$, as given by the manufacture is 0.97 . The throat diameter is $16.0 \mathrm{~mm}$ and the inlet and exit diameters are both $27.7 \mathrm{~mm}$. The impeller diameter is $114 \mathrm{~mm}$. The density of water is taken as $997 \mathrm{~kg} / \mathrm{m}^{3}$ and the viscosity is taken as 0.001 Pa.s based on the room temperature measurements.

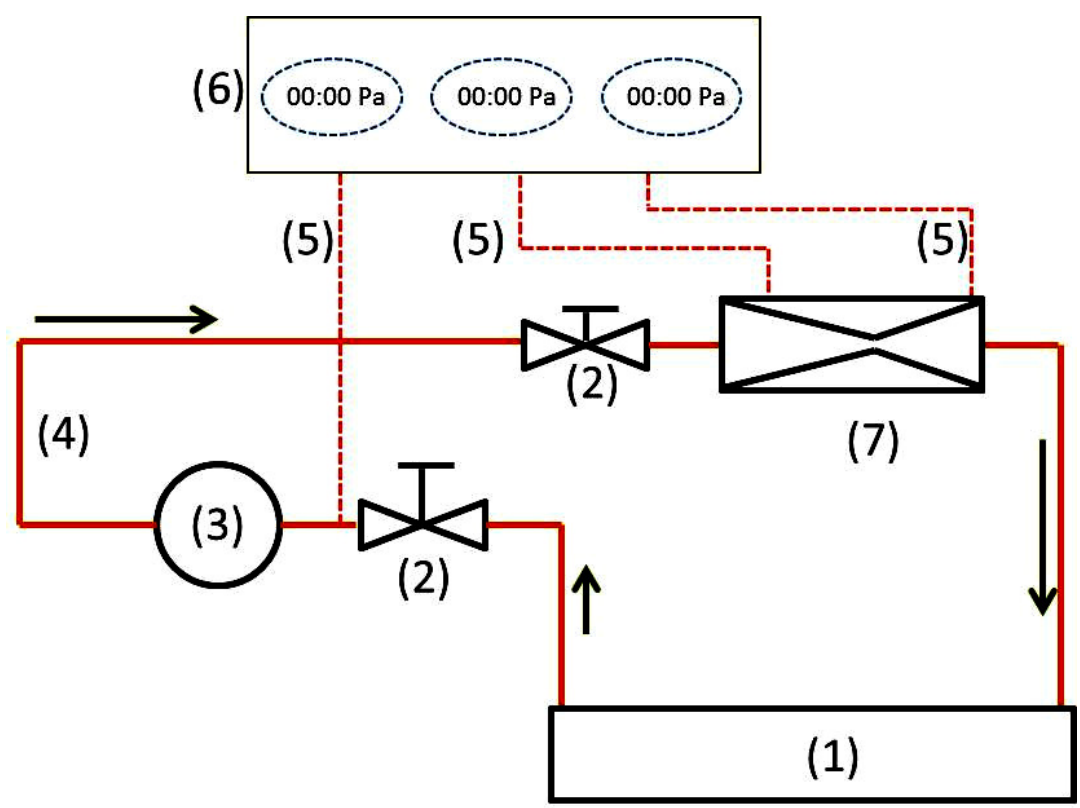

Figure 2: Line diagram representing the flow rig

(1) Water reservoir, (2) Valve, (3) Pump, (4) Water line, (5) Connection to transducer, (6) Electronic display and (7) Venturi meter 


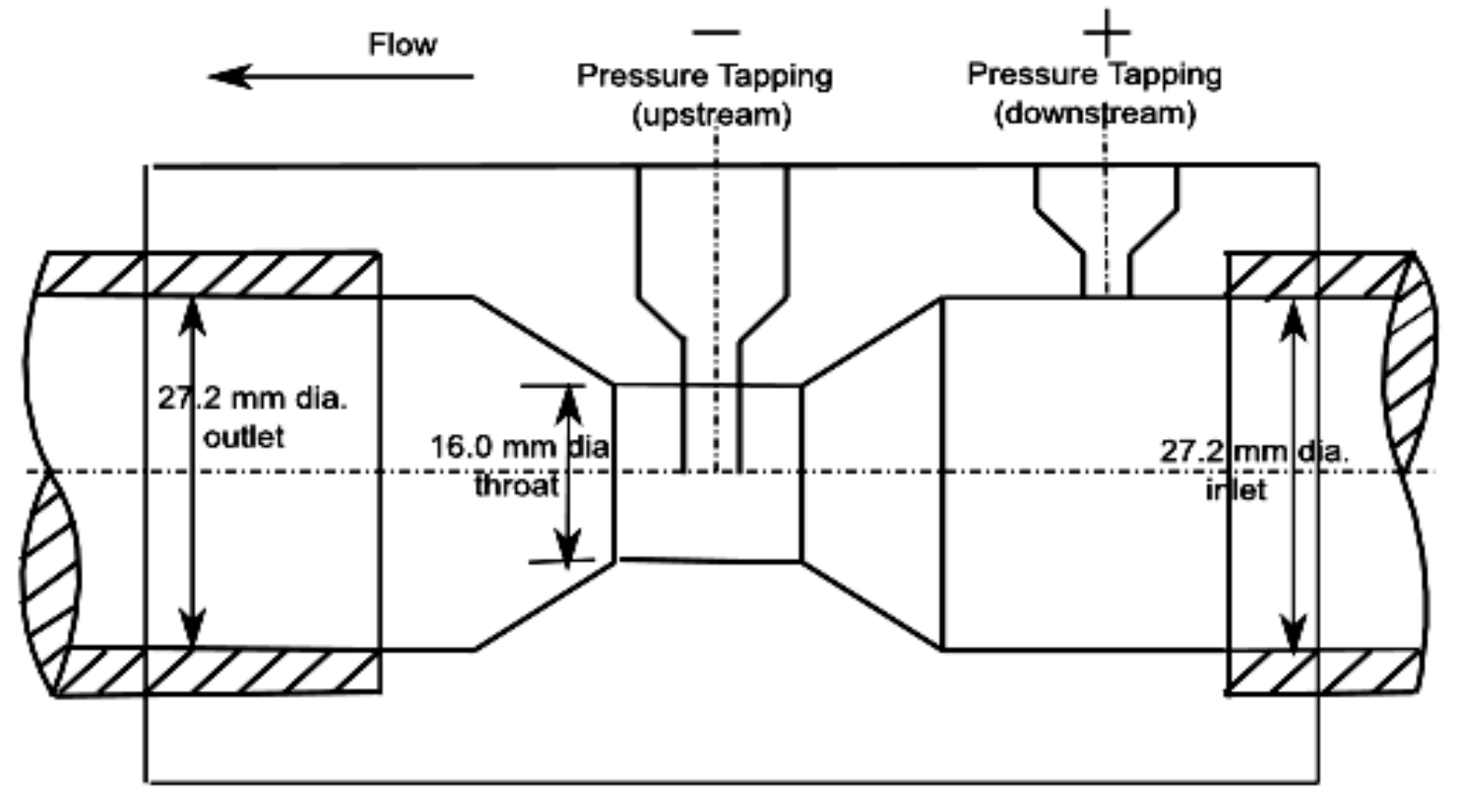

Coefficient of discharge, $\mathrm{Cd}=0.97$

Figure 3: The pump Venturi sectional view

\section{RESULTS}

The mean pressure drop across the Venturi for the series of tests for each speed is shown in Figure 4. The margin of fluctuations is also shown using error bars. These fluctuations are caused by cavitation effects as the pressure is reduced. The effect of cavitation on pressure drop is not much at $2000 \mathrm{rpm}$ and the instrument pressure limit is reached before much cavitation effects become pronounced. At 2500 and $3000 \mathrm{rpm}$, the effect of cavitation is well pronounced and reached before the instrument limits.

At low speed and where the effect of cavitation is minimal, the torque is much steady as can be seen in Figure 5. The cavitation causes the fluctuation of torque and power (Figure 6). All these three grow with increasing speeds as shown in Figures 4-6.

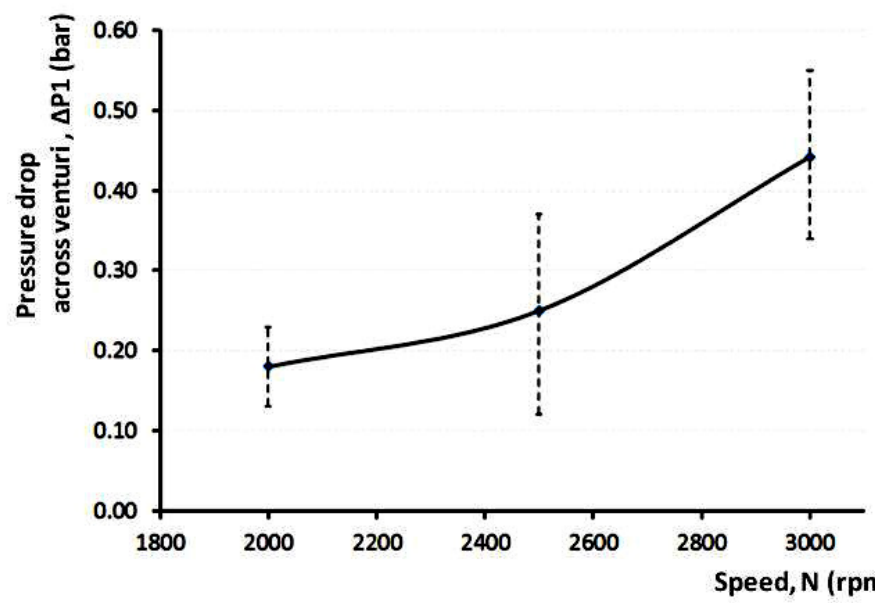

Figure 4: Pressure fluctuations across the Venturi throat

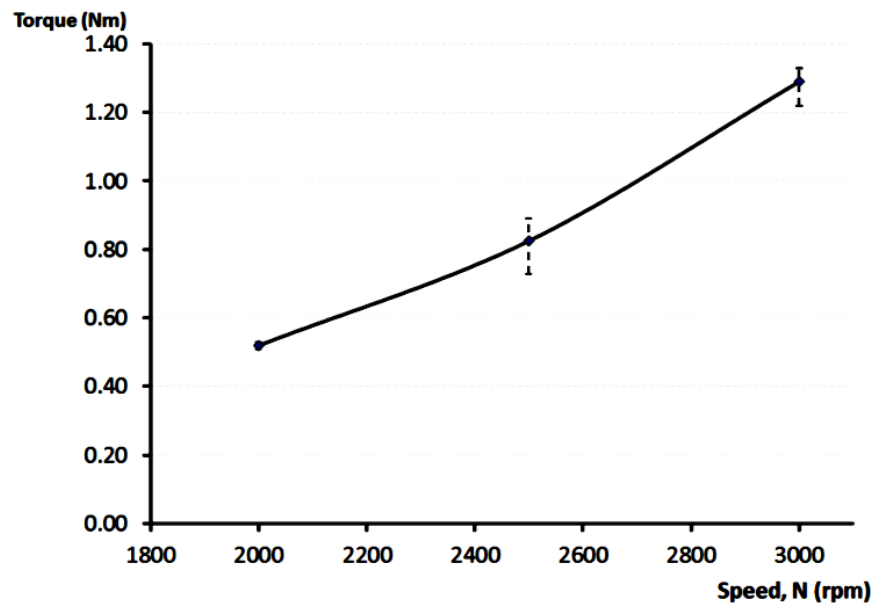

Figure 5: Mean torque versus speed

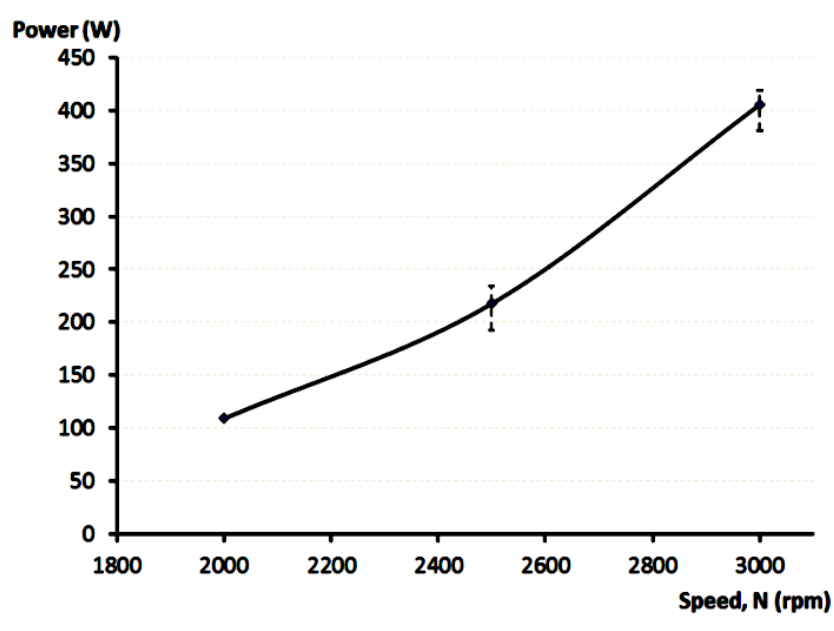

Figure 6: Power versus speed 
Figure 7 shows the performance chart of the H47 pump from the experiment. In this figure, the pump head is expressed in $\mathrm{kPa}$ so that it can be plotted on the same axis with Efficiency. The black solid curves show the pump head, the red lines are for the pump power and the dashed blue lines are the efficiencies at the 3 speeds. The speeds 3000, 2500 and 2000rpm correspond to Reynolds numbers of $4 \times 10^{7}, 3.4 \times 10^{7}$ and $2.7 \times 10^{7}$. All these three parameters increase with increasing Reynolds number.

The best efficiency of the pump is observed to be less than $45 \%$ overall but the best efficiency does not go with the highest Reynolds number. The pump performance in the experiment flow range is obtained with at 2500rpm and peaks at a flow rate of about $1.0 \mathrm{~L} / \mathrm{s}$ and pump head of $55.1 \mathrm{kPa}$ (or $5.6 \mathrm{~m}$ ) which requires a mechanical power of only about $85 \mathrm{~W}$.

A clearer performance of the pump is presented in non-dimensional form in Figure 8. This graph shows that the data collapse and can be fit. The maximum possible power coefficient is $2.48 \times 10^{-4}$ at a corresponding flow coefficient of $3.2 \times 10^{-3}$ giving a head coefficient of 0.078 at the best efficient point (BEP). The BEP is determined from the maxima of power coefficient curve, red in the Figure 8 corresponding to an efficiency of $42 \%$.

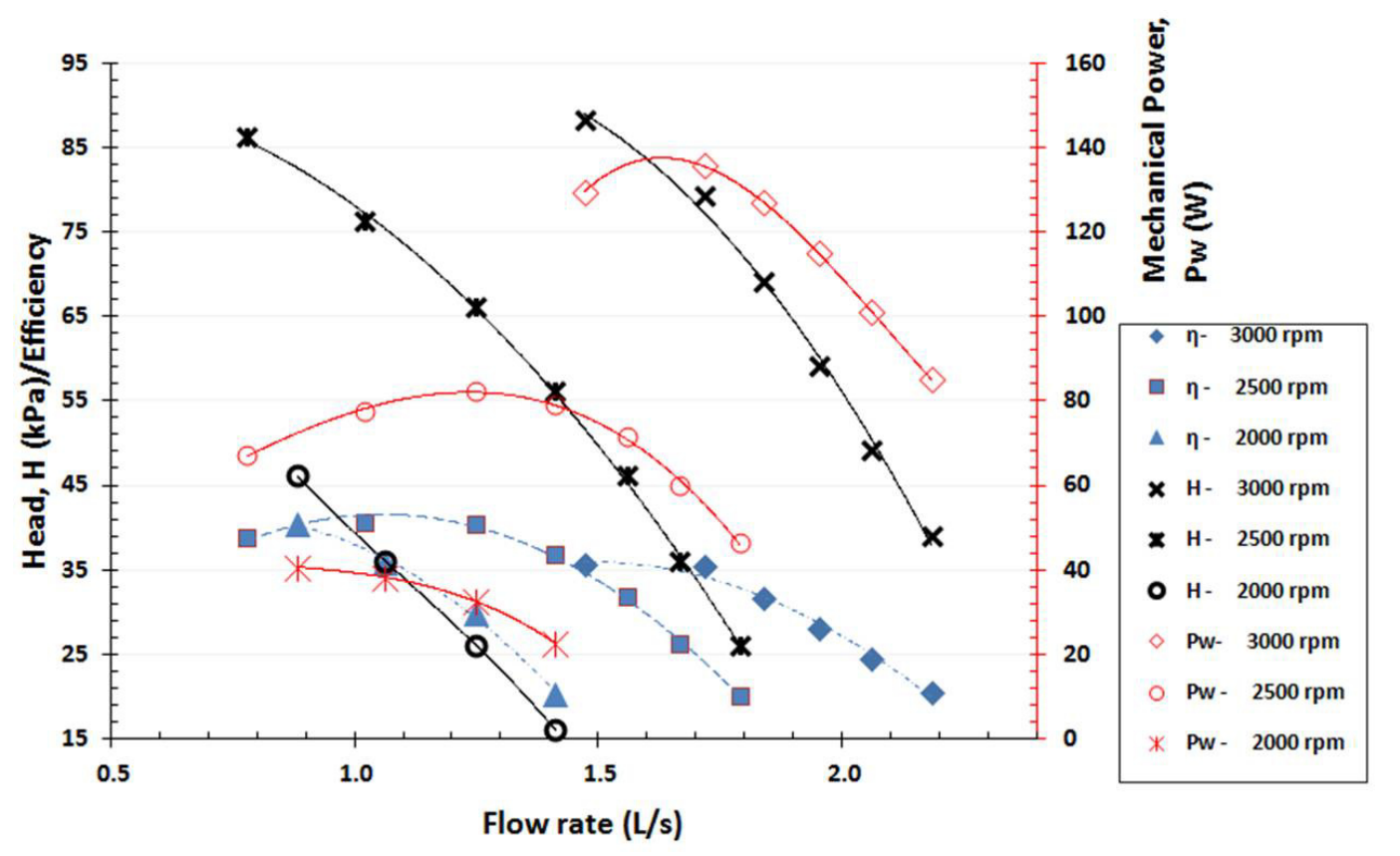

Figure 7: H47 Pump performance chart

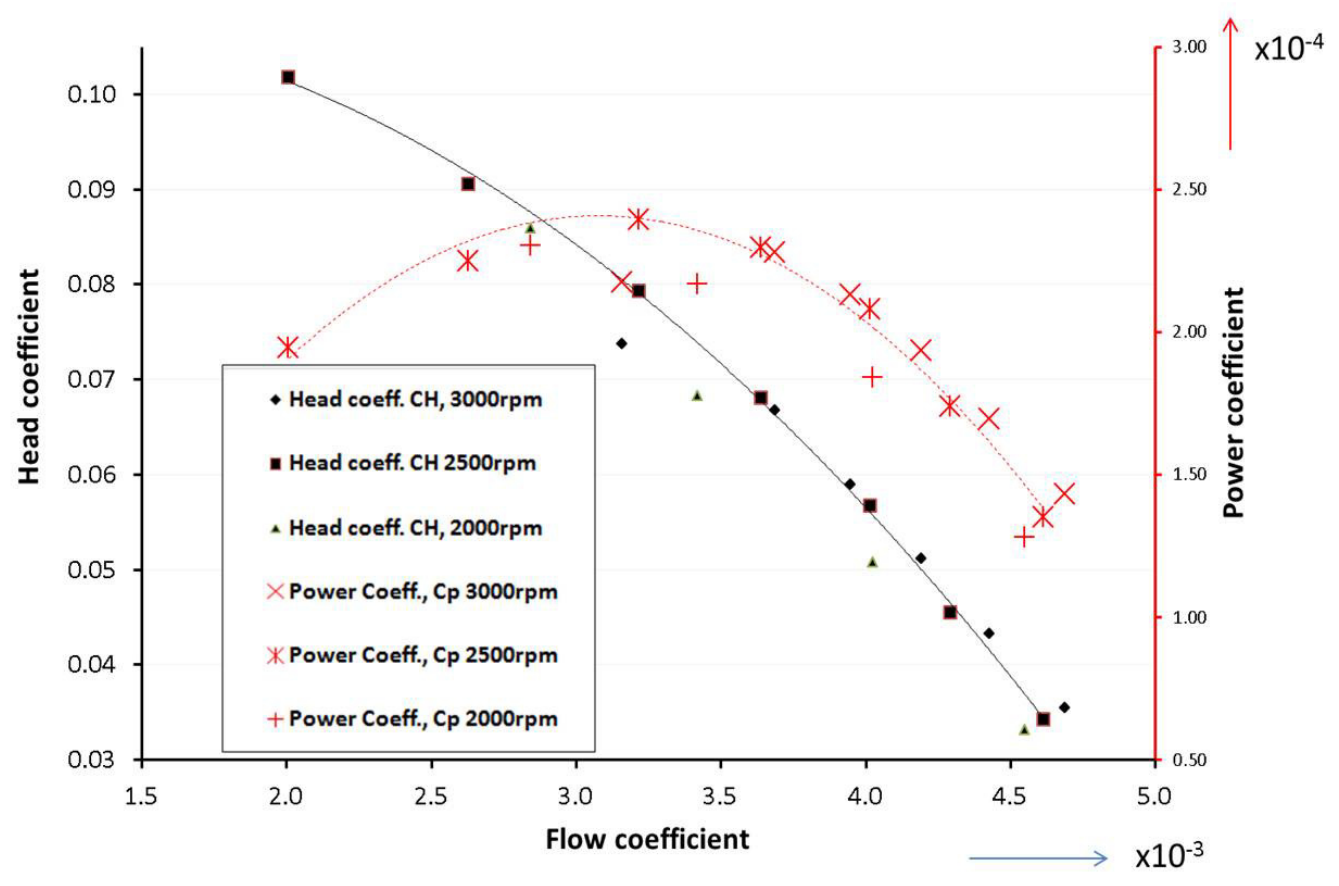

Figure 8: Non-dimensional form of pump performance 


\section{ANALYSIS AND DISCUSSION}

The head coefficient increases as the pump speed increases but peaks at the BEP. The onset of cavitation drops the pump heat and power coefficient. Given a pump with an impeller diameter twice the current one and operated at the BEP and using equations (9) to (12) will produce $9.93 \mathrm{~L} / \mathrm{s}$ with a pump head of $277.9 \mathrm{kPa}$ (or $28.4 \mathrm{~m}$ ) and requiring a mechanical power of $2.71 \mathrm{~kW}$.

\section{CONCLUSIONS}

A set of experiments to determine the performance of a pump was carried out at Reynolds number of $4 \times 10^{7}$, $3.4 \times 10^{7}$ and $2.7 \times 10^{7}$ respectively. The performance charts show that the maximum possible efficiency of the pump is less than $45 \%$ and the best efficiency point is around $42 \%$. The maximum possible power coefficient is $2.48 \times 10^{-4}$ at a corresponding flow coefficient of $3.2 \times 10^{-3}$ giving a head coefficient of 0.078 at the best efficient point (BEP). This pump can produce a head of $55.1 \mathrm{kPa}$ (or $5.6 \mathrm{~m}$ ). A nondimensional analysis was carried out on a hypothetical pump with twice impeller diameter. The flow rate is expected to be $9.93 \mathrm{~L} / \mathrm{s}$ and the pressure difference is expected to be $277.9 \mathrm{kPa}$ for the larger pump.

Volumetric flow can be increased by increasing the pumping power, impeller diameter or pumping speed. Since cavitation represents a loss of useful power and can be harmful to the blades, the choice of operating range should be away from the cavitation zone. The grove depth and the number of blades might also have effect on formation of cavitation and should be looked into in a further work.

\section{ACKNOWLEDGEMENT}

The authors wish to acknowledge Dr. K. Simmons and Dr. D. Hann the module convenors of TF2, University of Nottingham, UK, for their instruction manual. We are also grateful for the positive contributions from the peer reviewers from NIJOTECH.

\section{REFERENCES}

[1] R. Rayner, Pump Users Handbook, $4^{\text {th }}$ Edition, Oxford: Elsevier, 1995.

[2] Centrifugal Pump Test Set (H47), [Online] Available: http://www.tecquipment.com, [Accessed: 13 Nov. 2013].

[3] P. Timár "Dimensionless Characteristics of Centrifugal Pump, " in Proceeding of the 32nd International Conference of the Slovak Society of Chemical Engineering, Tatranské Matliare, 2005, pp.500-503.

[4] P.A. Ozor and S.N. Ojobor "Design, Construction and Measured Performance of a Single-Stage Centrifugal Pump Demonstration Unit" Nigerian Journal of Technology, Vol.31, No 3, pp.241-247, Nov. 2012.

[5] K.C. Thin, M.K Mya, and M.A Khin "Design and Performance Analysis of Centrifugal Pump." World Academy of Science, Engineering and Technology Vol. 46, pp. 422-429, 2008.

[6] J.F. Douglas, J.M Gasiorek and J.A Swaffield "Performance of Rotodynamic Machines" in Fluid Mechanics, 4th Ed., London: Pearson Education, 2001, pp. 741-762.

\section{NOMENCLATURE}

\begin{tabular}{cl}
\hline Variable & Quantity \\
\hline$A_{1}$ & Area of Inlet of the venturi $\left(\mathrm{m}^{2}\right)$ \\
$A_{2}$ & Throat area of the venturi $\left(\mathrm{m}^{2}\right)$ \\
$C_{d}$ & Coefficient of discharge \\
$D$ & Impeller diameter $(\mathrm{m})$ \\
$H$ & Pump total head $(\mathrm{m})$ \\
$L$ & Losses $\left(P-P_{W}\right)($ watts $)$ \\
$N$ & Pump speed $(\mathrm{rpm})$ \\
$P$ & Power input to pump $($ watts $)$ \\
$P_{w}$ & Hydraulic power of pump $($ watts $)$ \\
$p_{o}$ & Pump outlet static pressure $\left(N / \mathrm{m}^{2}\right)$ \\
$\Delta p$ & Pressure drop across the venturi $\left(N / \mathrm{m}^{2}\right)$ \\
$\eta$ & Efficiency of pump $(\%)$ \\
$\mu$ & Viscosity (N/ms) \\
$\rho$ & Density of the liquid $\left(\mathrm{kg} / \mathrm{m}^{3}\right)$ \\
$\omega$ & Pump speed $(\mathrm{rad} / \mathrm{s})$ \\
\hline
\end{tabular}

